\title{
Quantitative Control over Electrodeposition of Silica films onto Single-Walled Carbon Nanotube Surfaces
}

\author{
Mandakini Kanungo ${ }^{1}$, Hugh S. Isaacs ${ }^{1}$, and Stanislaus S. Wong ${ }^{1,2, *}$ \\ ${ }^{1}$ Condensed Matter Physics and Materials Sciences Department, Brookhaven National \\ Laboratory; Building 480; Upton, NY 11973 \\ ${ }^{2}$ Department of Chemistry; State University of New York at Stony Brook; Stony Brook, NY \\ 11794-3400 \\ * To whom correspondence should be addressed. Phone: 631-632-1703; 631-344-3178. \\ E-mail: sswong@notes.cc.sunysb.edu; sswong@bnl.gov
}




\section{Mechanism of base-catalyzed hydrolysis and associated sol-gel reaction used in electrodeposition procedures:}

It has been shown from the literature that the reduction of oxygen can follow one of two possible pathways. The first proceeds through a four-electron reduction process in which oxygen is reduced to $\mathrm{OH}^{-}$ions, as shown in Equation 1.1-4

$$
\mathrm{O}_{2}+2 \mathrm{H}_{2} \mathrm{O}+4 \mathrm{e}^{-} \rightarrow 4 \mathrm{OH}^{-}
$$

The second is associated with a two-electron reduction process in which the oxygen is reduced to hydrogen peroxide and hydroxide ions (Equation 2).

$$
\mathrm{O}_{2}+2 \mathrm{H}_{2} \mathrm{O}+2 \mathrm{e}^{-} \rightarrow \mathrm{H}_{2} \mathrm{O}_{2}+2 \mathrm{OH}^{-}
$$

The presence of $\mathrm{OH}^{-}$will increase the $\mathrm{pH}$ at the electrode surface, which in turn will initiate a base-catalyzed condensation reaction of the hydrolyzed tetramethoxysilane. The base-catalyzed condensation mechanism can be described as follows (Eqns. 3 \& 4). ${ }^{4}$

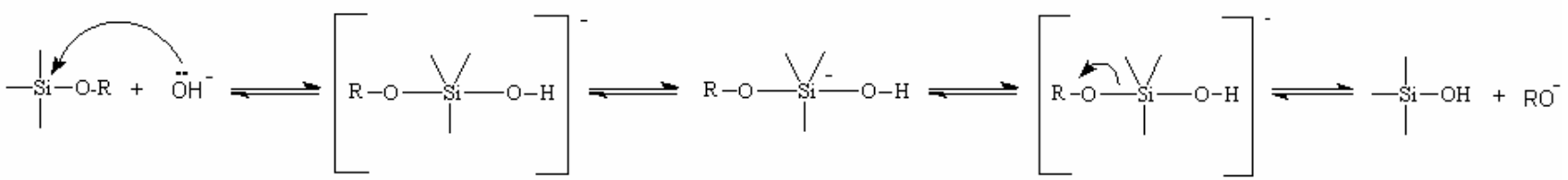

[3]

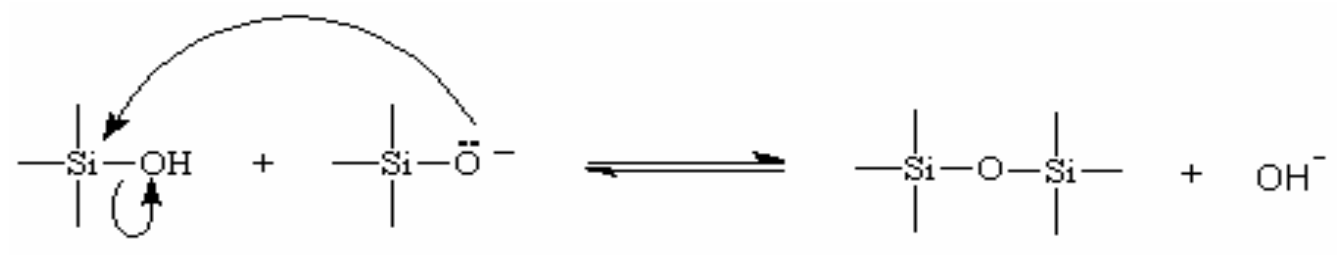




\section{Supplementary Figure-1}
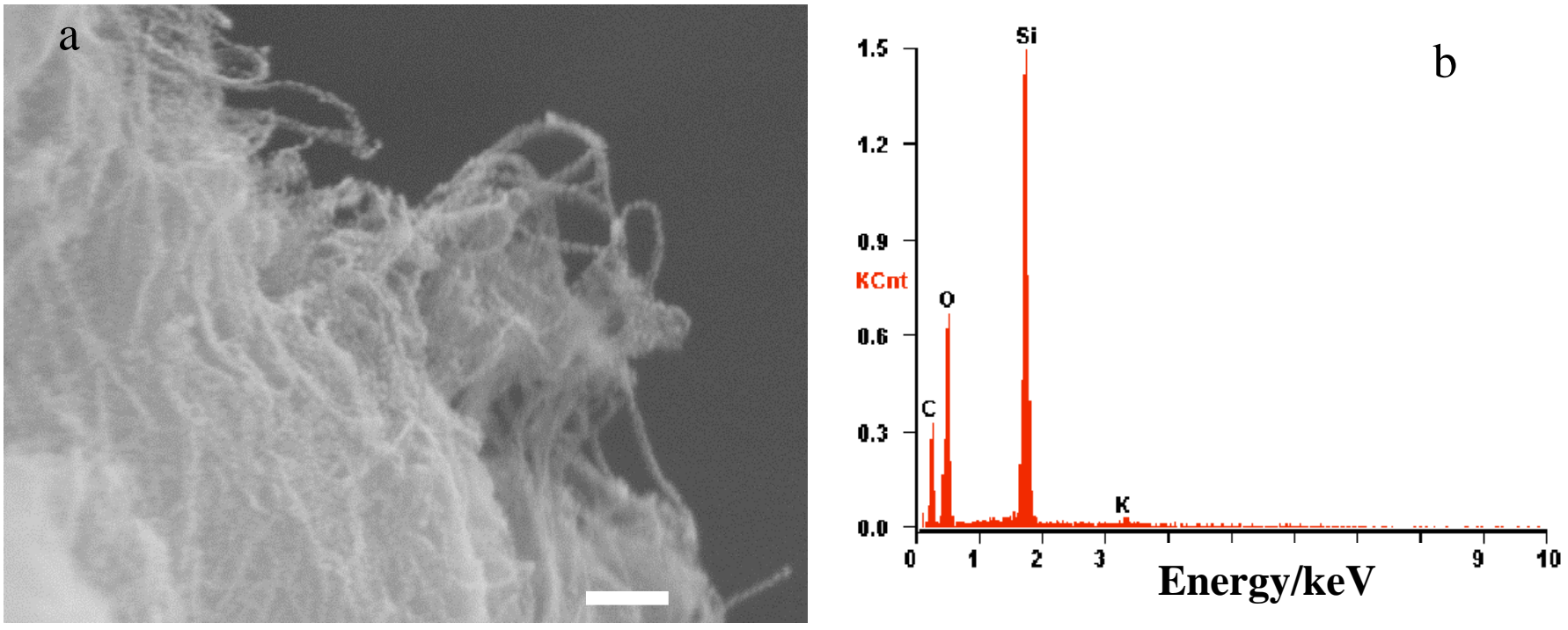

Supplementary Figure S1. SEM image and the corresponding EDS spectrum of carbon nanotubes deposited on a platinum foil electrode along with silica. The scale bar in part 'a' is $100 \mathrm{~nm}$. 

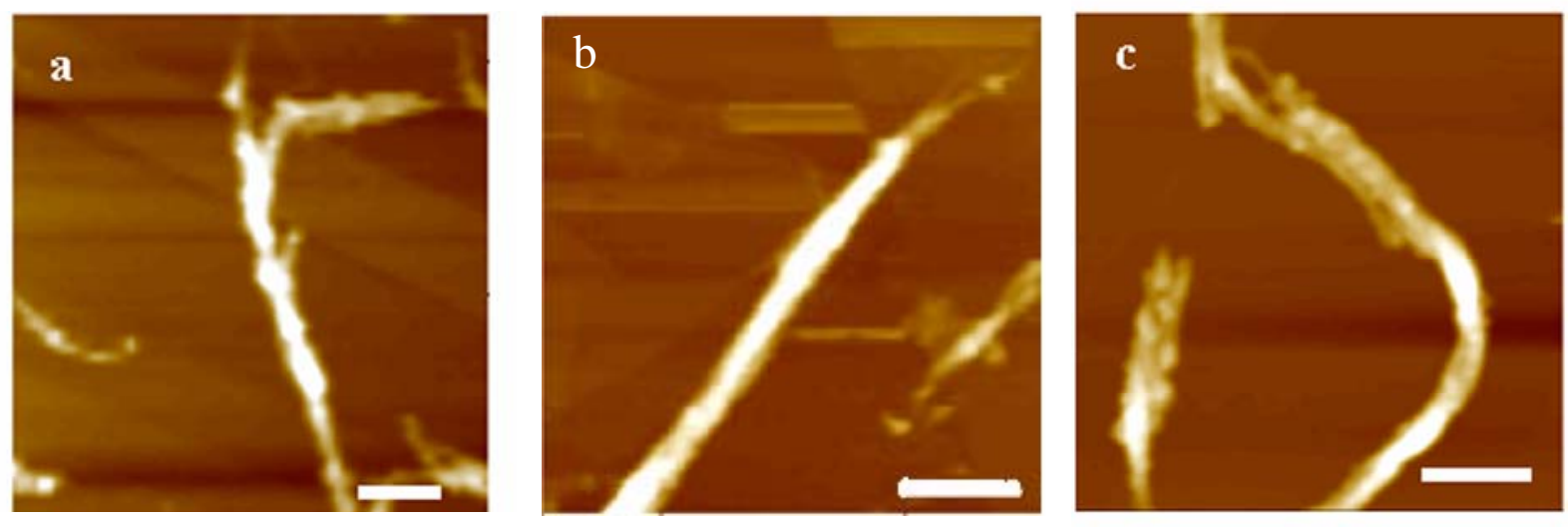

Supplementary Figure S2. (a-c) AFM height images of silica-coated nanotubes synthesized by electrochemical deposition of carbon nanotubes dispersed in the solution. The potential applied was $-800 \mathrm{mV}$ and the concentration of TMOS used in these images was $7.4 \cdot 10^{-5} \mathrm{M}, 2.92 \cdot 10^{-4} \mathrm{M}$, and $5.6 \cdot 10^{-4} \mathrm{M}$ respectively. The $\mathrm{z}$ scale data is $50 \mathrm{~nm}$ for (a), $100 \mathrm{~nm}$ for (b), and $300 \mathrm{~nm}$ for (c). Scale bars are $100 \mathrm{~nm}$ for (a), $200 \mathrm{~nm}$ for (b), and 250 nm for (c), respectively. 


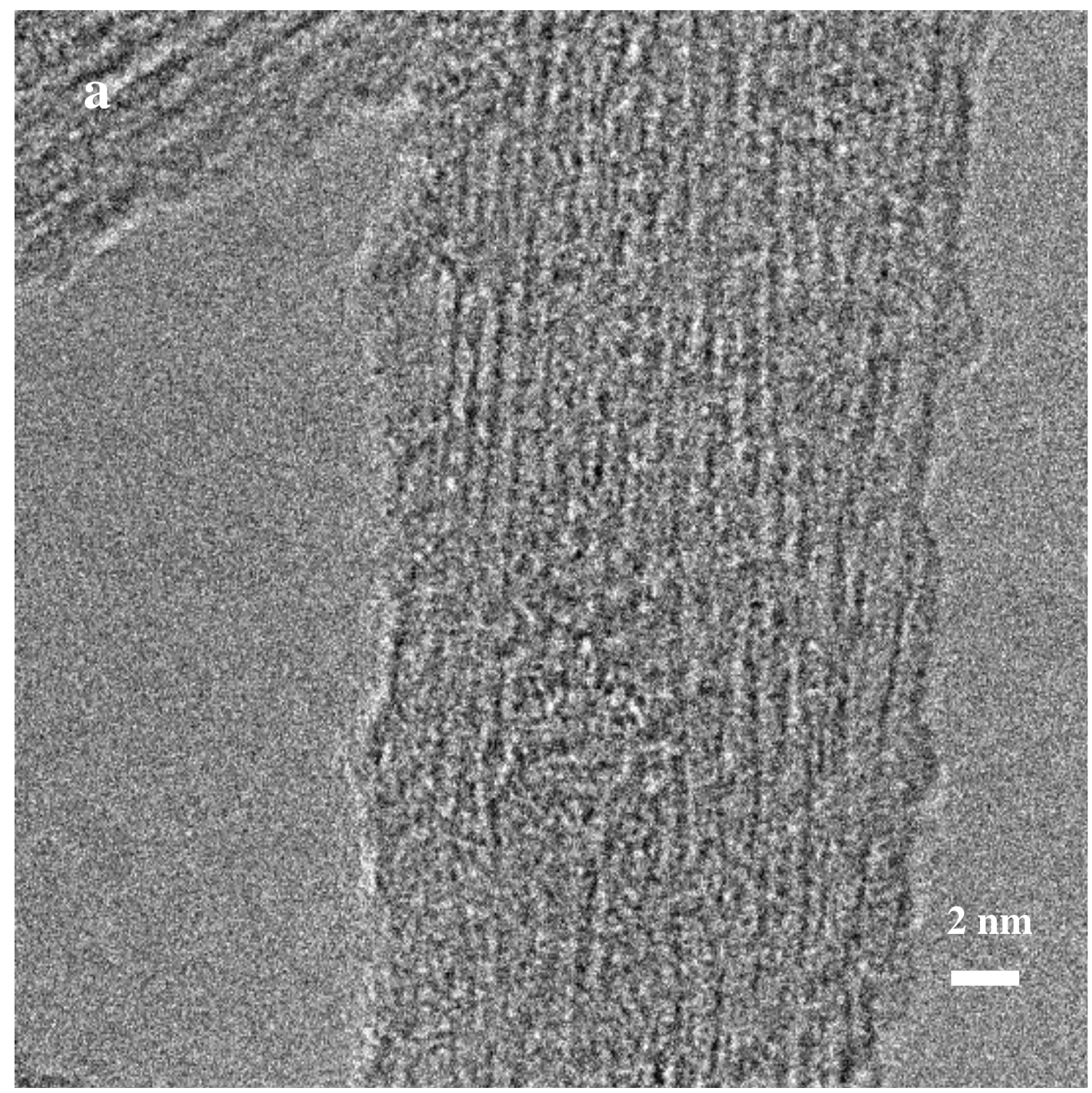




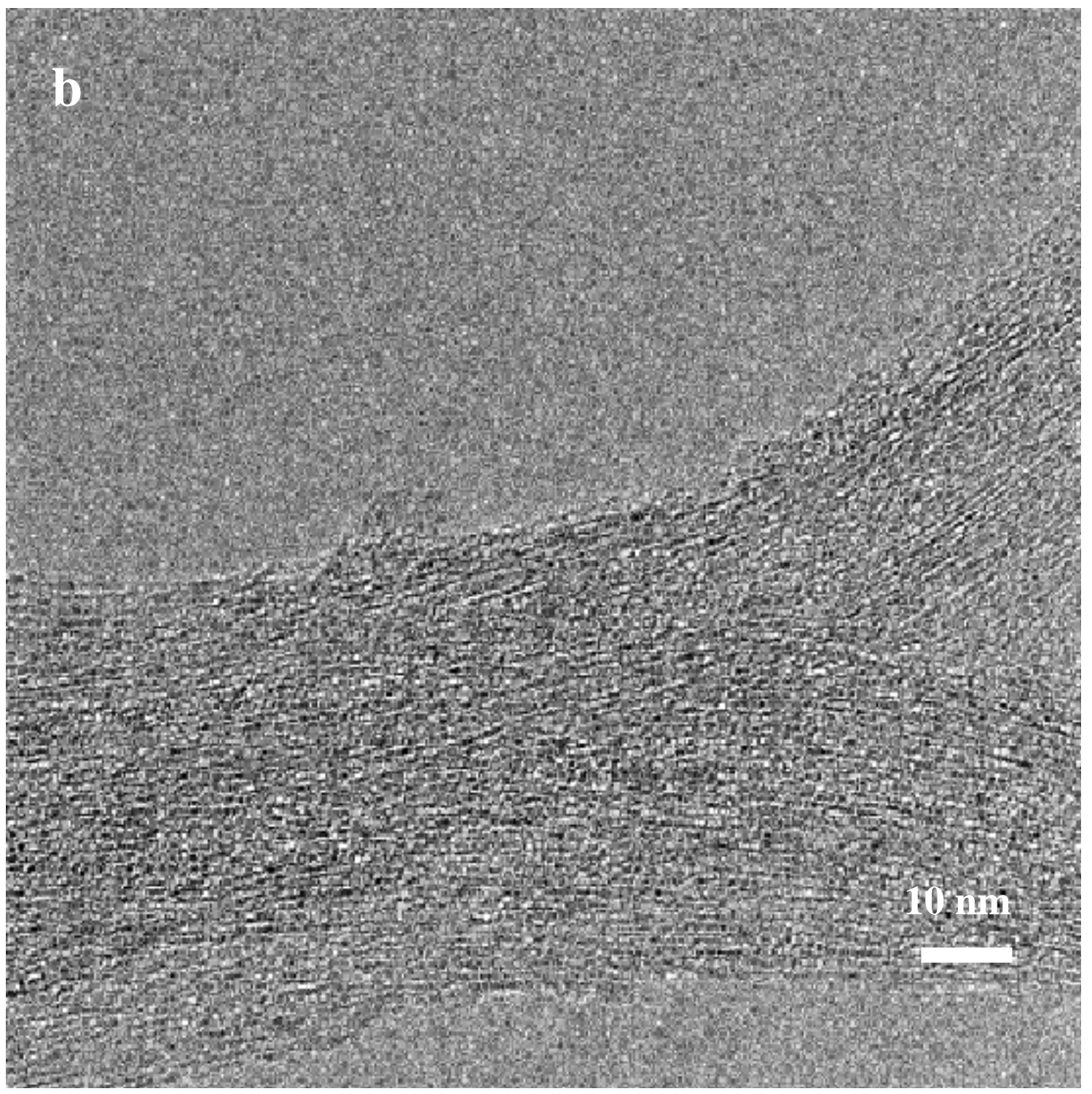

Supplementary Figure S3: (a) HRTEM image of Si-SWNT-1 electrodeposited at -600 mV. (b) HRTEM image of Si-SWNT-2 electrodeposited at $-700 \mathrm{mV}$. 


\section{Supplementary Figure-4}
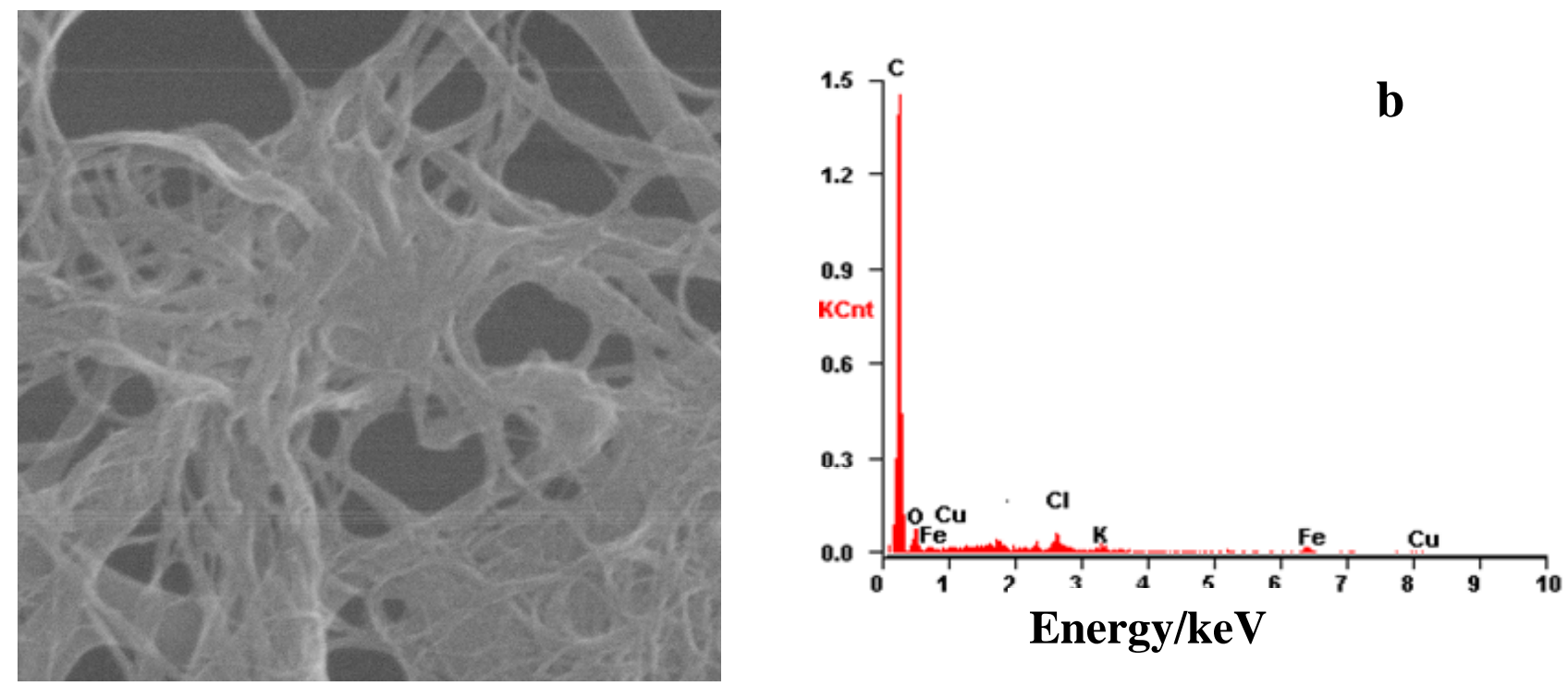

Supplementary Figure S4. (a) SEM image of a control sample (Si-SWNT-ctrl-1) and (b) the corresponding EDS spectrum associated with this image 


\section{Supplementary Figure -5}
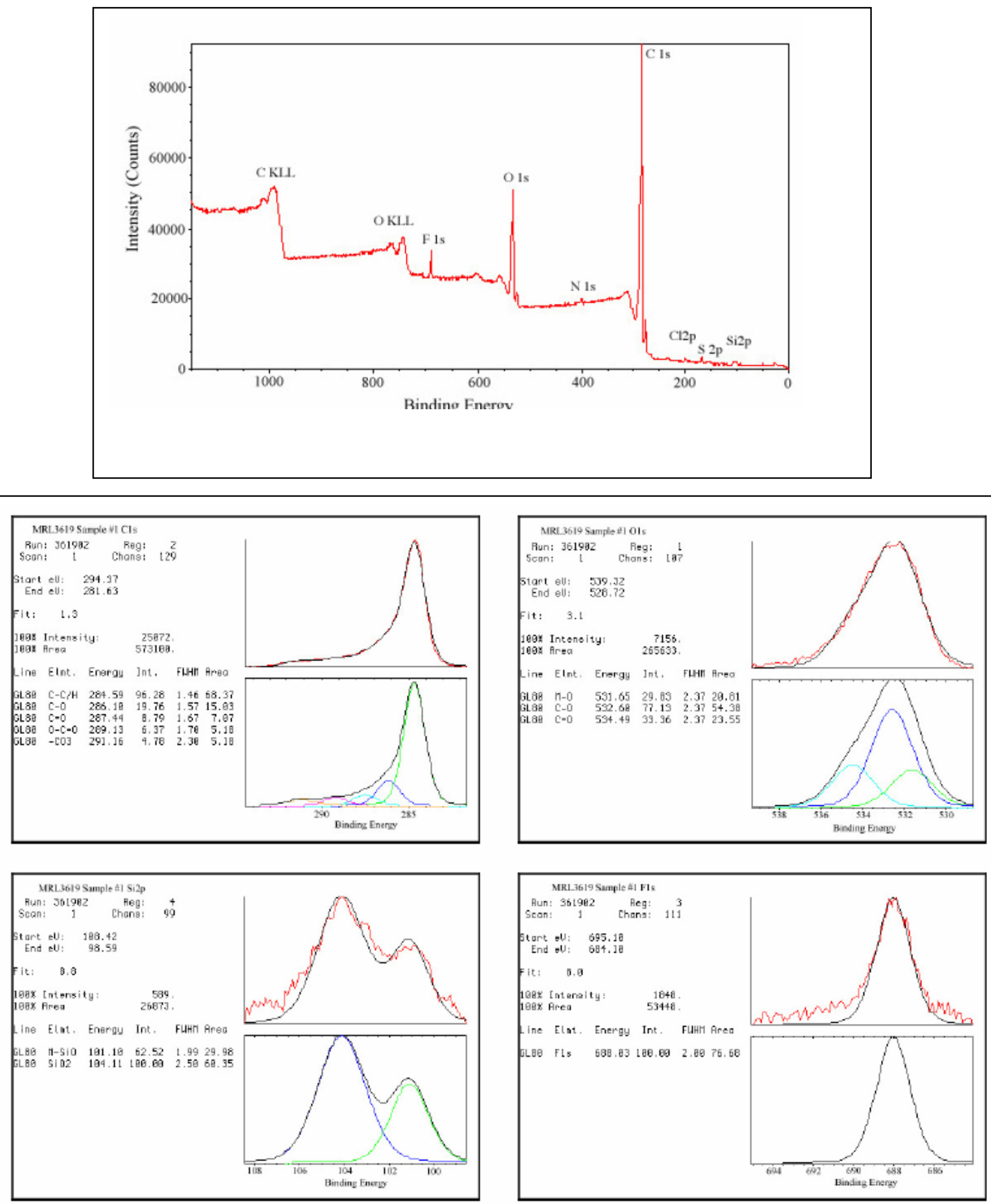


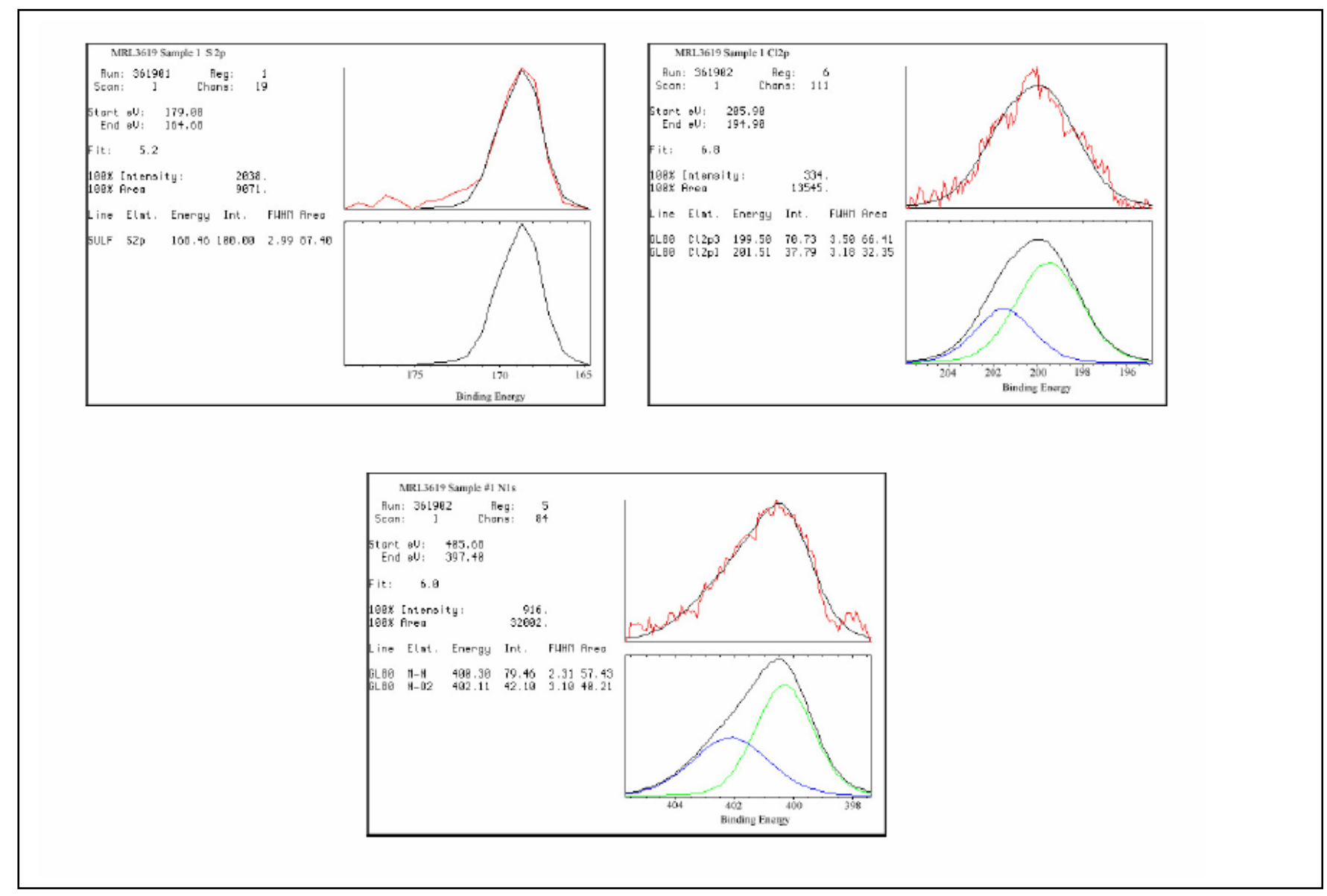

Supplementary Figure S5. High-resolution XPS spectra of purified single-walled carbon nanotubes. 


\section{Supplementary Figure -6}
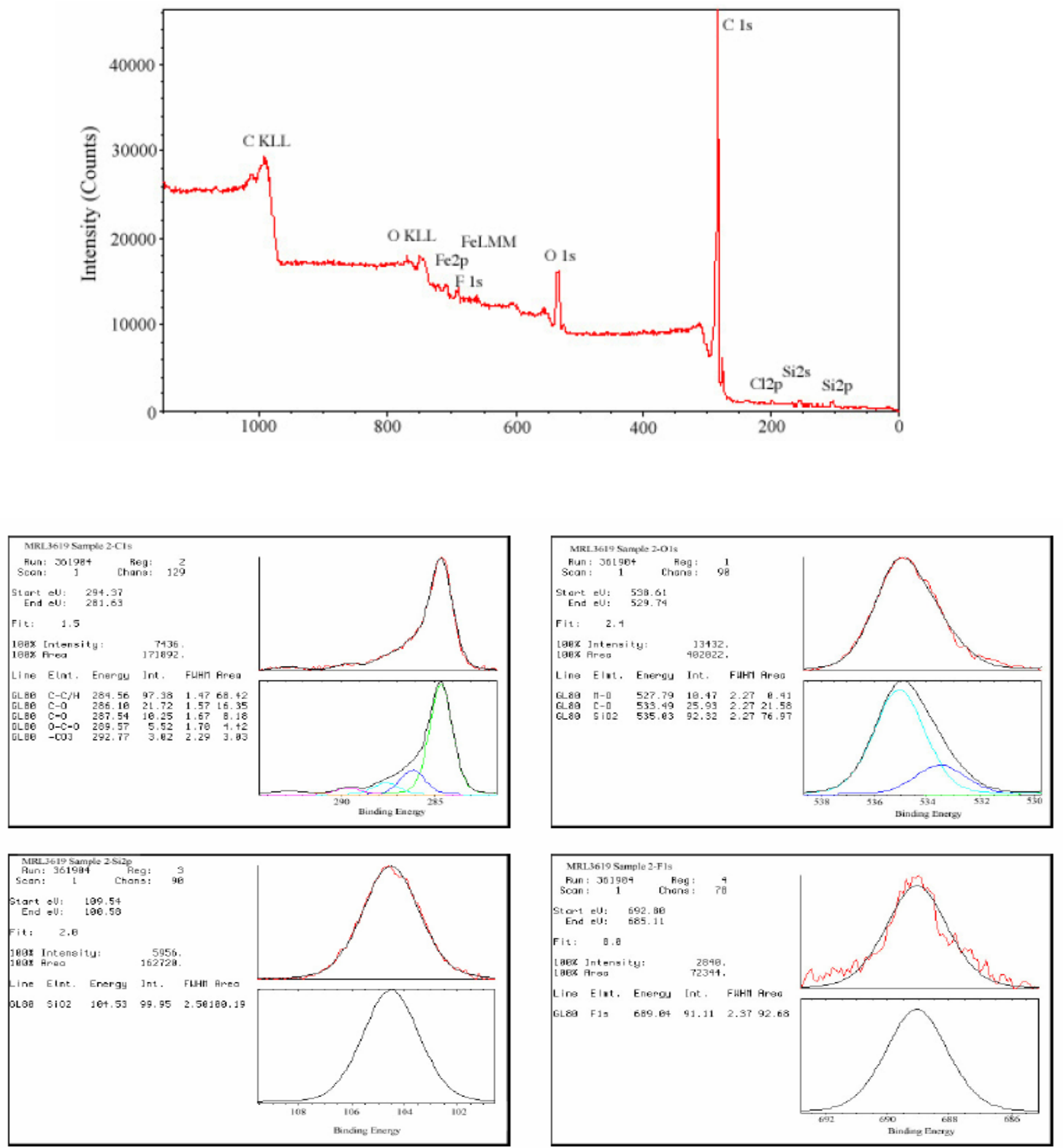


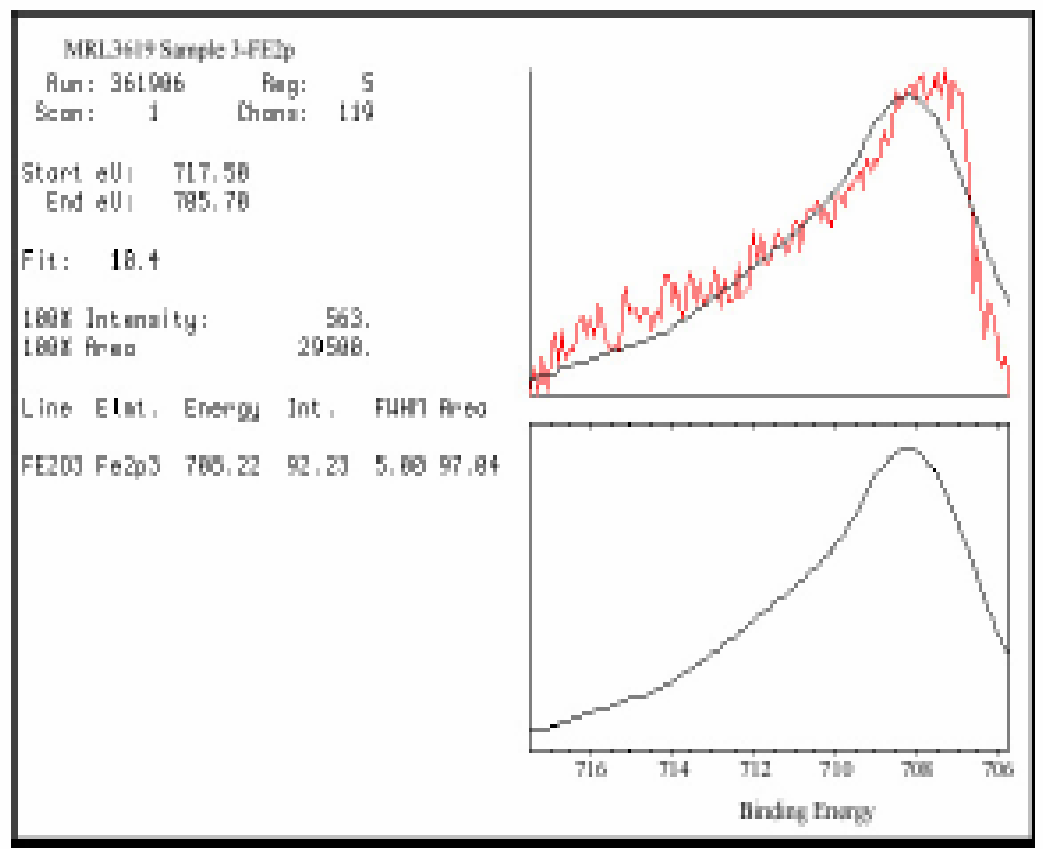

Supplementary Figure S6. High-resolution XPS spectra of a control sample (Si-SWNT-ctrl-1). 


\section{Supplementary Figure- 7}
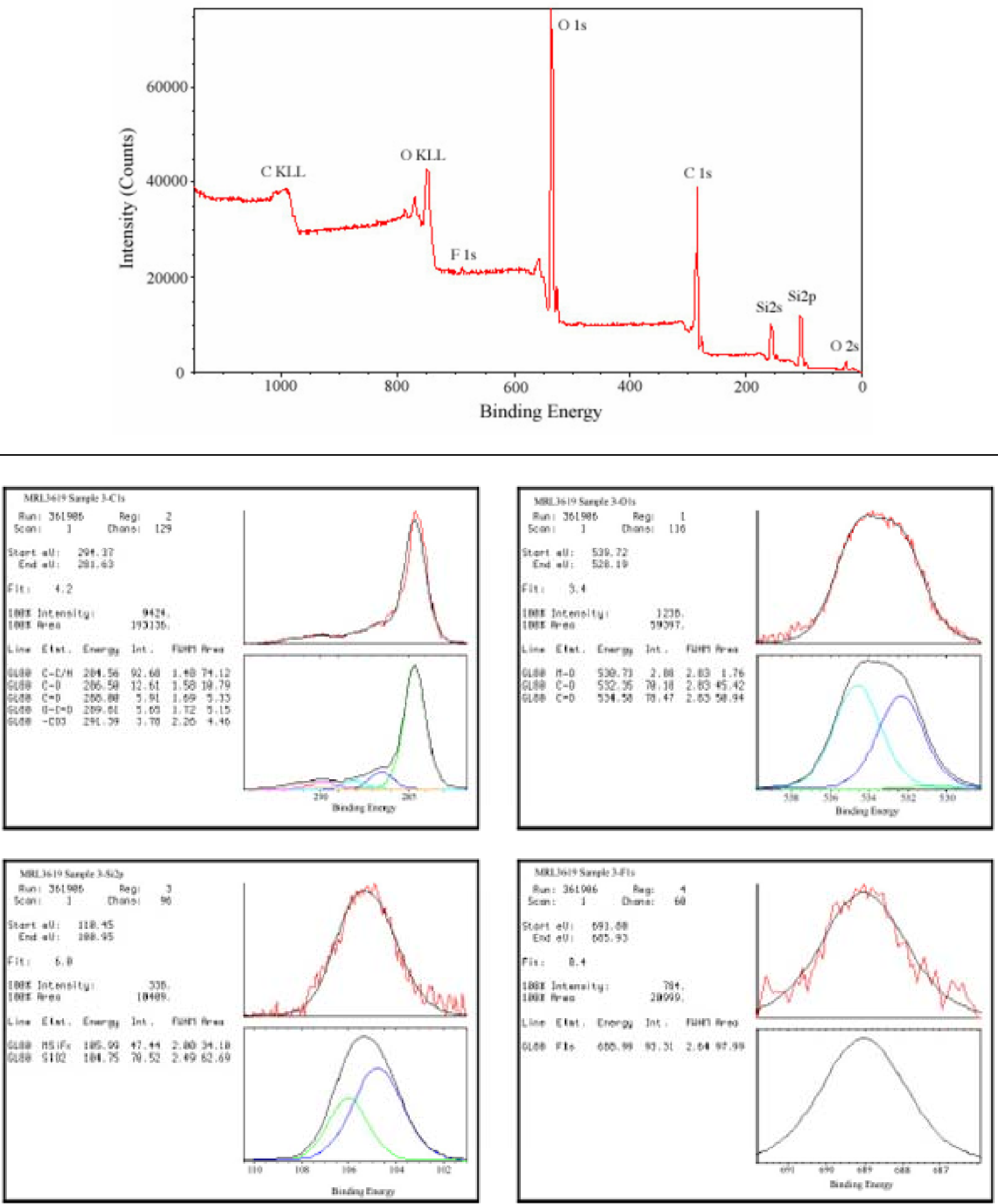

Supplementary Figure S7. High-resolution XPS spectra of silica-coated nanotubes (Si-SWNT-1). 


\section{References}

(1) Bard, A. J.; Faulkner, I. R. Electrochemical Methods. Fundamentals and Applications New York, 1980.

(2) Bockris, J. O. M.; Khan, S. U. M. Surface Electrochemistry New York, 1993.

(3) Aldykiewicz Jr., A. J.; Davenport, A. J.; Isaacs, H. S., J. Electrochem. Soc., 1996, 143, 147.

(4) Iler, R. K. The Chemistry of Silica New York, 1979. 